\title{
Quantitative high-throughput optical sizing of individual colloidal nanoparticles by wide-field imaging extinction microscopy
}

Lukas M. Payne, Attilio Zilli, Yisu Wang, Wolfgang Langbein, Paola Borri

Lukas M. Payne, Attilio Zilli, Yisu Wang, Wolfgang Langbein, Paola Borri, "Quantitative high-throughput optical sizing of individual colloidal nanoparticles by wide-field imaging extinction microscopy ," Proc. SPIE 10892, Colloidal Nanoparticles for Biomedical Applications XIV, 108920J (7 March 2019); doi: 10.1117/12.2507632

SPIE. Event: SPIE BiOS, 2019, San Francisco, California, United States 


\title{
Quantitative high-throughput optical sizing of individual colloidal nanoparticles by wide-field imaging extinction microscopy - The "long shadow" effect
}

\author{
Lukas M. Payne ${ }^{\mathrm{a}}$, Attilio Zilli ${ }^{\mathrm{a}}$, Yisu Wang ${ }^{\mathrm{a}}$, Wolfgang Langbein ${ }^{\mathrm{b}}$, and Paola Borri ${ }^{\mathrm{a}}$ \\ ${ }^{a}$ Cardiff University School of Biosciences, Museum Avenue, Cardiff CF10 3AX, United \\ Kingdom \\ ${ }^{b}$ Cardiff University School of Physics and Astronomy, The Parade, Cardiff CF24 3AA, United \\ Kingdom
}

\begin{abstract}
We present a wide-field imaging technique recently developed by us to measure quantitatively the optical extinction cross section $\sigma_{\text {ext }}$ of individual nanoparticles. The technique is simple, high speed, and enables the simultaneous acquisition of hundreds of nanoparticles in the wide-field image for statistical analysis, with a sensitivity corresponding to the detection of a single gold nanoparticle down to $2 \mathrm{~nm}$ diameter. Notably, the method is applicable to any nanoparticle (dielectric, semiconducting, metallic), and can be easily and cost-effectively implemented on a conventional wide-field microscope. Of specific significance for accurate quantification, we show that $\sigma_{\text {ext }}$ depends on the numerical aperture of the microscope illumination due to the oblique incidence, even for spherical particles in an isotropic environment. This "long shadow" effect needs to be taken into account when comparing $\sigma_{\text {ext }}$ to theoretical values calculated under plane wave illumination at normal incidence. Owing to the accurate experimental quantification of $\sigma_{\text {ext }}$, one can then use it to determine the nanoparticle size, as demonstrated here on gold nanoparticles of $30 \mathrm{~nm}$ nominal diameter. This technique thus has the potential to become a simple and cost-effective new tool for accurate size characterization of single small nanoparticles, complementing time-consuming and expensive methods such as electron microscopy.
\end{abstract}

Keywords: single particle analysis, optical microscopy, extinction cross section

\section{INTRODUCTION}

Colloidal nanoparticles (NPs) are widely utilised for many applications ranging from optoelectronic devices exploiting quantum confinement at the nanoscale, ${ }^{1}$ to drug delivery, ${ }^{2}$ in-vitro diagnostics and sensing. ${ }^{3}$ Of specific interest are their optical properties which offer many opportunities in light-based minimally- or noninvasive imaging and sensing. Owing to inhomogeneities in NP size, shape, and composition, the properties of an individual NP can significantly differ from the ensemble average, hence increasing attention has been devoted recently to developing methods capable of detecting single NPs. Yet, it is important to detect and characterize a large number of single NPs, for statistical significance.

It is well known that NPs exhibit linear optical properties described by an absorption cross section, $\sigma_{\text {abs }}$, an elastic scattering cross section, $\sigma_{\text {sca }}$, and the resulting extinction cross section, $\sigma_{\text {ext }}=\sigma_{\text {abs }}+\sigma_{\text {sca }}$ (where we assume that inelastic scattering is negligible). The scaling of $\sigma_{\text {ext }} \propto R^{\gamma}$ for spherical particles of radius $\mathrm{R}$ is known from Mie theory in the Rayleigh regime, for wavelengths much smaller than the NP size. ${ }^{4}$ In this regime, one can use the dipole approximation resulting in $\gamma=3$ for small NPs, where the extinction is dominated by absorption, and increasing towards $\gamma=6$ for larger NPs, when scattering has a significant effect.

We have recently developed a technique that measures the optical extinction cross-section $\sigma_{\text {ext }}$ of individual nanoparticles using wide-field transmission microscopy. ${ }^{5-7}$ It enables simultaneous acquisition of hundreds of nanoparticles for statistical analysis. The method is simple, rapid, and quantitative. Using rapid referencing, fast acquisition, and a deconvolution analysis, we have demonstrated a shot-noise limited sensitivity down to

Further author information: Send correspondence to borrip@cf.ac.uk

Colloidal Nanoparticles for Biomedical Applications XIV, edited by Marek Osiński, Wolfgang J. Parak, Proc. of SPIE Vol. 10892, 108920J · C 2019 SPIE · CCC code: 1605-7422/19/\$18 · doi: 10.1117/12.2507632 
$0.4 \mathrm{~nm}^{2}$. Measurements on a set of individual gold nanoparticles of $5 \mathrm{~nm}$ diameter using this method gave $\sigma_{\text {ext }}=(10.0 \pm 3.1) \mathrm{nm}^{2}$, consistent with theoretical expectations, and well above the background fluctuations of $0.9 \mathrm{~nm}^{2}$ which set the detection limit to a $2 \mathrm{~nm}$ diameter gold NP. Notably, the method is applicable to any NP (including dielectric or semiconducting), and can be easily and cost-effectively implemented on a conventional wide-field microscope.

In this paper, we describe our more recent efforts toward accurately measuring the magnitude of $\sigma_{\text {ext }}$, taking into account the geometry of the illumination. When imaging with a microscope objective lens of high numerical aperture (NA) to achieve high spatial resolution and sensitivity, the illumination for brightfield imaging typically extends over an NA range from zero to the objective NA, and for darkfield imaging over an NA range above the objective NA, including highly oblique incident angles. We show here that this gives rise to a measured $\sigma_{\text {ext }}$ larger than the value expected under plane wave illumination at normal incidence. Intuitively speaking, $\sigma_{\text {ext }}$ is the shadow cast by the NP, i.e. the light missing in transmission geometry upon absorption and scattering from the NP. The larger cross-section can be understood as originating from the longer shadow cast by the NP onto the imaged sample plane for oblique illumination, which we call the "long shadow" effect. Notably, albeit apparently obvious, this effect has not been discussed in the literature so far.

\section{EXPERIMENT}

Ultra-uniform spherical gold nanoparticles (GNP)s of nominally $30 \mathrm{~nm}$ and $50 \mathrm{~nm}$ size were obtained from Nanocomposix, with distributions in diameter, determined from transmission electron microscopy (TEM), given by, $D=29.4 \pm 1.3 \mathrm{~nm}$ and $D=49.4 \pm 2.2 \mathrm{~nm}$. Particles were spin-coated onto a glass coverslip (refractive index $n=1.52$ ), cleaned using a procedure involving successive dips/washes in toluene, acetone, hydrogen peroxide, and water. The sample was immersed in silicone oil (refractive index $n=1.52$ ), attached to a glass slide $(n=1.52)$, and sealed with clear nail varnish. The measurements were performed on a Nikon Ti-U inverted microscope, with a $1.34 \mathrm{NA}$ oil condenser and 1.45 NA (100x) oil objective (Nikon lambda series), or 0.95 (40x) dry objective (Nikon lambda series), as previously described. ${ }^{5,7}$ Here, the oil objective was used for the illumination NA-dependence measurements discussed below, while the dry objective was used to obtain a wider field of view and hence increase the number of measured GNPs for improved statistics for particle sizing. Thorlabs bandpass filters of $40 \mathrm{~nm}$ bandwidth and center wavelengths $450 \mathrm{~nm}$ (B) and $550 \mathrm{~nm}(\mathrm{G})$, were used to filter the white-light illumination (Nikon Ti-DH, D-LH/LC, $100 \mathrm{~W}$ tungsten lamp). Images were acquired with a scientific-CMOS camera (PCO Edge 5.5), with full well capacity $N_{\mathrm{fw}}=30000$ photoelectrons capable of 100 frames per second (FPS) at full frame $(2560 \times 2160$ pixels $)$. We used a cropped sensor size of $2048 \times 768$ pixels, with a 270 FPS framerate. The NA range of the condenser was manually controlled by the condenser aperture diaphragm, and home-built dark-field disks. A total of 6144 frames were captured in brightfield for each of the illumination NA ranges used, with exposure time and lamp intensity set to keep the maximum intensity in the field of view below the non-linear response of the sensor (about 60000 counts of the 16-bit digitizer range). The total count of 6144 frames were made up of a capture sequence in the form of $N_{r}=24$ repetitions of $j=128$ exposures with the sample in focus at a position, $P_{1}$, and $j=128$ exposures of the sample in focus at a lateral position, $P_{2}$, shifted by a distance $s$ relative to $P_{1}$. The shifted frames act as reference for those at $P_{1}$. Note that $s$ must be chosen taking into account the wavelength of illumination and the numerical aperture of the collection (i.e. is dependent on the objective and filter used), which determine the diameter of the point spread function (PSF). Typically, $s \leq 2.1 \mu \mathrm{m}$ for the specific objectives and filter ranges described, with minimum $s$ limited by the overlap of measured areas of the PSFs of particles and their shifted images. Maximum $s$ is not fundamentally limited, except by practical considerations, such as particle density, image size, etc. Since the extinction image in general may be expressed as $\Delta=1-I / I_{0}$, with $I$ transmitted intensity in the presence of the particle and $I_{0}$ the corresponding reference intensity (i.e. a differential transmission image),$^{5}$ we can develop $\Delta_{1}$ from the above sequence for $P_{1}$ as,

$$
\Delta_{1}=\frac{1}{N_{r}} \sum_{N_{r}}\left\{1-\frac{\sum_{j} I_{\mathrm{P}_{1}}}{\sum_{j} I_{\mathrm{P}_{2}}}\right\} .
$$

The same expression holds for $\Delta_{2}$ with the particle in $P_{2}$. 
(a)

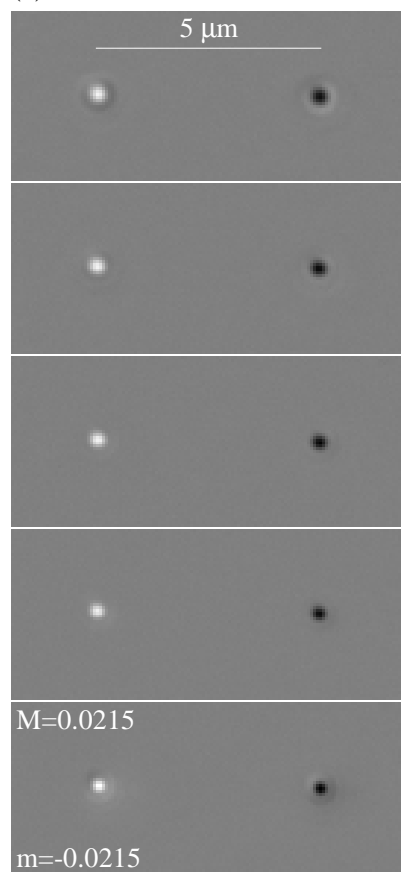

30nm diameter GNP

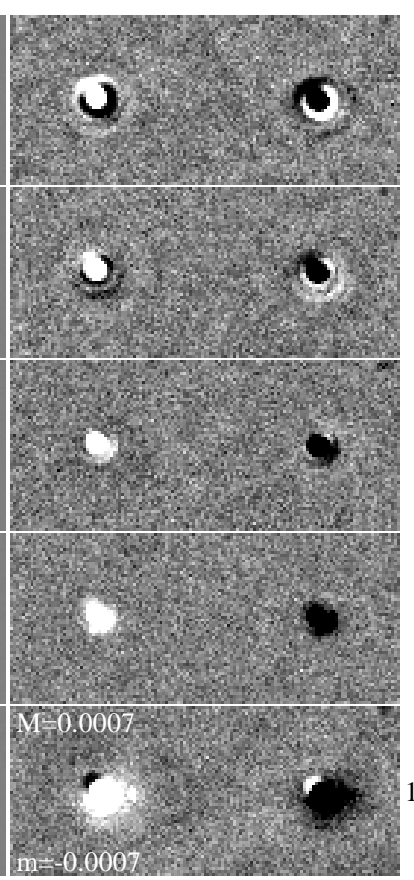

(b)

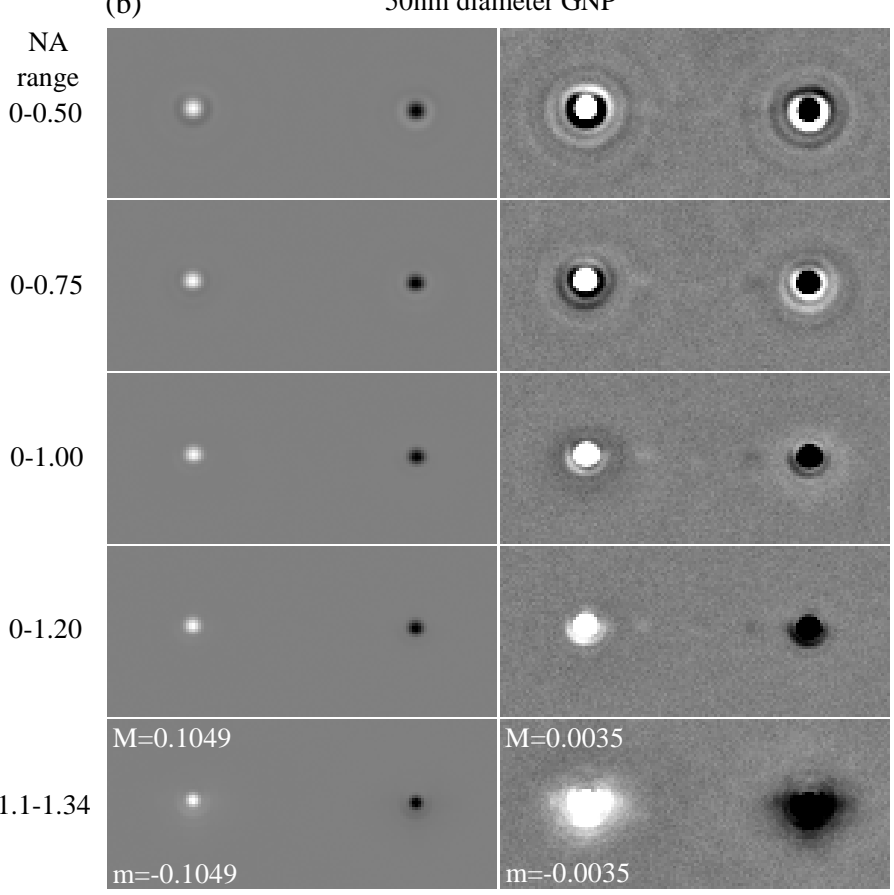

Figure 1. Spatially cropped differential transmission image $\Delta_{1}$ of single GNPs of $30 \mathrm{~nm}$ (a) and $50 \mathrm{~nm}$ (b) diameter for various illumination NA ranges as indicated, using the color bandpass filter G and a shift of $5 \mu \mathrm{m}$ (see text). Data are given on two greyscale ranges from $\mathrm{m}$ (black) to $\mathrm{M}$ (white) as indicated, with one (left columns) covering the full data ranges, and the other (right columns) covering about $3 \%$ of the full range, to reveal weak features including the shot noise.

\section{RESULTS AND DISCUSSION}

The extinction cross-section is defined to be

$$
\sigma_{\text {ext }}=\frac{P_{\text {ext }}}{I_{0}},
$$

with $P_{\text {ext }}$ the power extinguished by the particle from the incident field, and $I_{0}$ the incident field intensity. Thus, we can determine the cross-section of an individual particle within the image by integrating the intensity of $\Delta$ within an area, $A_{\mathrm{i}}$, of radius, $r_{\mathrm{i}}$, around the particle. Details of this procedure are given in Ref. 5,7 . In those works, we typically used $r_{i} \approx 3 \lambda /(2 \mathrm{NA})$, and matched condenser and objective NA, which collected most of the cross-section, up to a residual estimated to be below $10 \%$. Using a larger $r_{i}$ increases the noise and collects more of the disturbing influence of nearby structures in the sample. However, a point of note is that the radial dependence of $\Delta_{1}$ will depend on the illumination NA range, described by the minimum NA, and the maximum $\overline{\mathrm{NA}}$, due to the partially coherent brightfield imaging. The NA range determines the spatial coherence of the illumination in the image plane, and thus the extent to which interferences between scattered and transmitted fields play a role. Importantly, such field interferences can have a much larger extension than the intensity point-spread function, and can lead to significant systematic errors in the evaluation of $\sigma_{\text {ext }}$ at finite $r_{i}$.

We investigated the effect of the illumination spatial coherence by measuring the same GNPs with different illumination NA ranges, as shown in Fig. 1 for the filter G. In order to allow the analysis of larger $r_{i}$, we used here a shift of $s=5 \mu \mathrm{m}$. In the data for the $50 \mathrm{~nm}$ GNP, which has a higher signal to noise ratio, and also a stronger contribution of $\sigma_{\text {sca }}$, rings affected by the spatial coherence of the illumination are clearly visible, specifically for the smallest investigated illumination NA range of 0 to 0.5 . Notably, these rings change their radial dependence with NA range, being most suppressed for the largest range shown, 0 to 1.2. Using an annular range 1.1 to 1.34, typically employed for dark-field imaging in conjunction with an $0.95 \mathrm{NA}$ objective, ${ }^{5}$ shows a wider extension of the rings, and also significant deviation from a circular shape, which we attribute to optical aberrations of objective and condenser. 

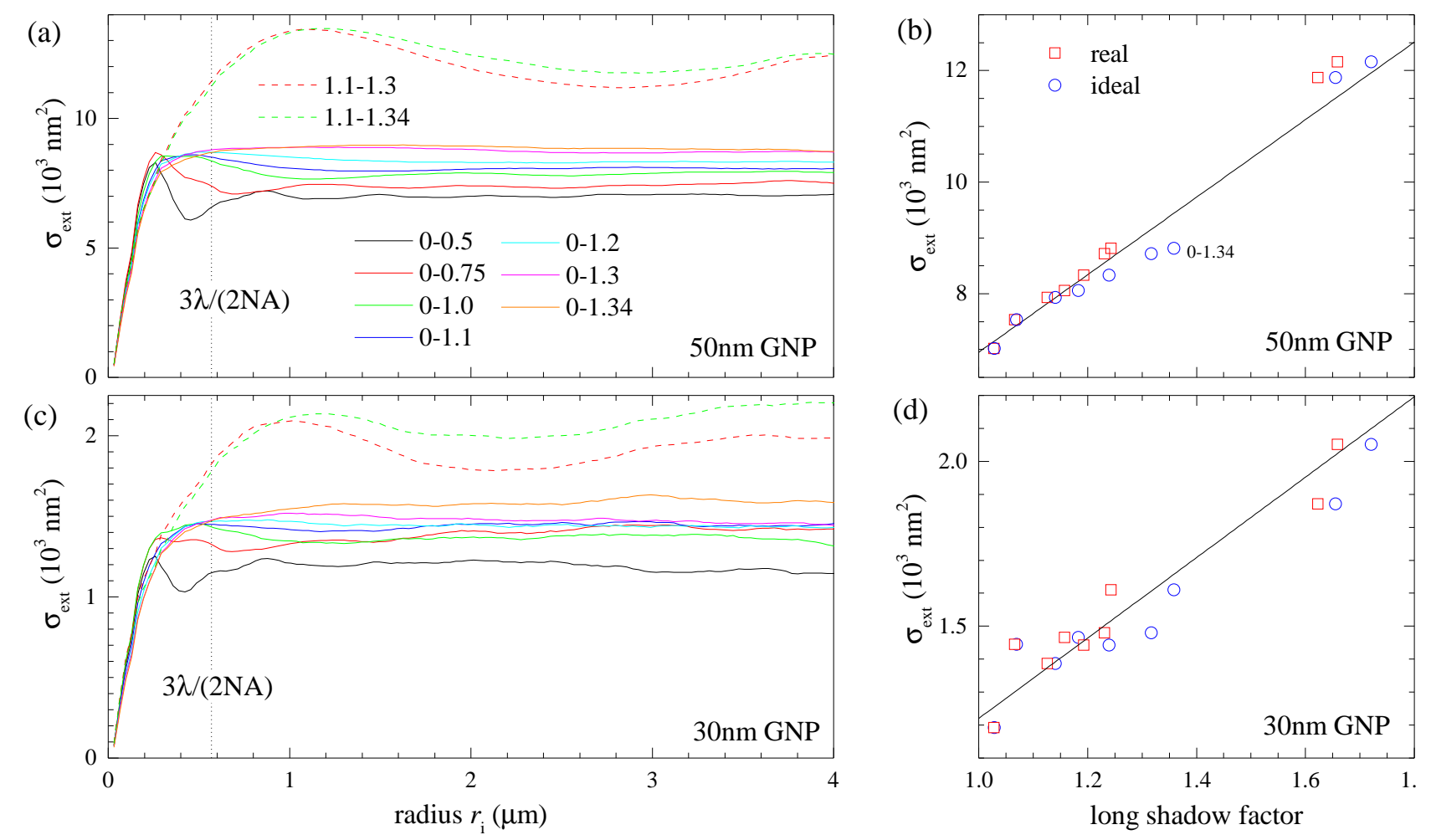

Figure 2. Extinction cross-section $\sigma_{\text {ext }}$ for a GNP of $50 \mathrm{~nm}$ diameter. (a) $\sigma_{\text {ext }}$ versus the radius $r_{i}$ of analysis, for different illumination NA ranges, as labelled. (b) Measured extinction cross-section (limit value of $\sigma_{\text {ext }}$ for large $r_{i}$ ) versus long shadow factor. The line shows the long shadow prediction. Blue circles use the ideal long shadow factor, while red squares take into account the NA dependent illumination intensity (see text). (c),(d) as (a),(b), but for a GNP of 30 nm diameter.

The extinction cross-section analysis of the data shown in Fig. 1 is given in Fig. 2. Let us first look at the data for the $50 \mathrm{~nm}$ GNP in (a), as they have a higher signal to noise ratio. For the disk-shaped illumination ranges with $\underline{\mathrm{NA}}=0$, we observe that the oscillations with $r_{i}$ are becoming less pronounced and of lower amplitude with increasing $\overline{\mathrm{NA}}$. At the same time, the limit value of $\sigma_{\text {ext }}$ for large $r_{i}$ is increasing. At $r_{i} \approx 3 \lambda /(2 \mathrm{NA})$, the result for the largest $\overline{\mathrm{NA}}$ used is well converged, confirming that it is a good choice for an illumination NA range matching the objective range. Instead, for the annular illumination ranges, with $\mathrm{NA}=1.1$, the oscillations are strong and extended over a large spatial range, evidence of a long illumination spatial coherence. Furthermore, the visually extrapolated $\sigma_{\text {ext }}$ is about $70 \%$ higher than for small NA.

Similar results are found for the $30 \mathrm{~nm}$ GNP in Fig. 1c, but showing more influence of background structure, and slightly different interference fringes, possibly due to the lower importance of scattering, resulting in a phase shift of the scattered wave and thus a phase shift of the interference pattern.

To discuss the effect of the spatial coherence of the illumination, we show in Fig. 3a the first order spatial coherence $C(\mathbf{d})=\left\langle E(\mathbf{r}) E^{*}(\mathbf{r}+\mathbf{d})\right\rangle_{\mathbf{r}} /\left\langle|E(\mathbf{r})|^{2}\right\rangle_{\mathbf{r}}$, with the shift $\mathbf{d}$, introduced by the NA range. We calculated $C(\mathbf{d})$ in scalar approximation as the Fourier-transform of the two-dimensional wavevector range corresponding to the NA range at $550 \mathrm{~nm}$ wavelength, assuming constant intensity in the condenser back focal plane. Note that $C(\mathbf{d})$ only depends on the distance $|\mathbf{d}|$ and not on the direction of $\mathbf{d}$ due to the axial symmetry of the wavevector range. We can see that for $\underline{\mathrm{NA}}=0$ the extension of $C(\mathbf{d})$ scales inversely with $\overline{\mathrm{NA}}$. For the annular ranges instead, $C(\mathbf{d})$ shows a fast and a slow oscillation, and a larger extension. The fast oscillation is averaged by the detection PSF, while the slow oscillation remains, resulting in the oscillation of $\sigma_{\text {ext }}$ with $1-2 \mu \mathrm{m}$ period observed in Fig. 2. To exemplify the effect of the illumination coherence, we show in Fig. 3b the analyzed $\sigma_{\text {ext }}$ for a dielectric scatterer. We see that the analyzed $\sigma_{\text {ext }}$ oscillates around zero, reflecting the constructive and destructive interference of the scattered field with the illumination. Again, the length scale of the oscillations 

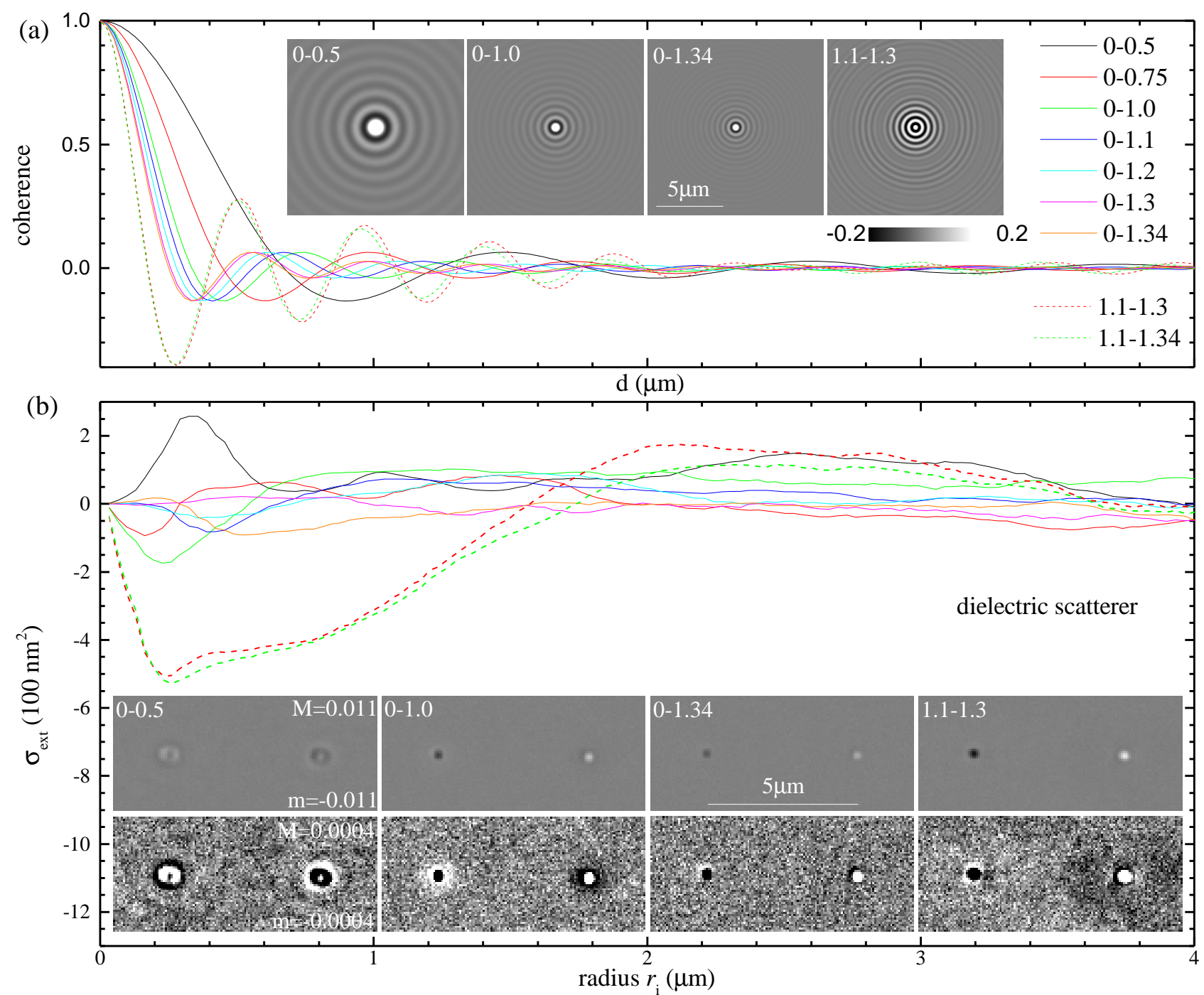

Figure 3. (a) First-order spatial coherence $C(\mathbf{d})$ of the illumination versus $|\mathbf{d}|$ for different illumination NA ranges, as labelled (see text). Insets show corresponding $C(\mathbf{d})$ on a greyscale as given. (b) Extinction cross-section $\sigma_{\text {ext }}$ for a dielectric scatterer versus the radius $r_{i}$ of analysis, for different illumination NA ranges, as labelled. The images show the corresponding $\Delta_{1}$, for NA ranges as indicated. Data are given on two greyscale ranges from $\mathrm{m}$ (black) to M (white) as indicated, with the top row covering the full data range, and the bottom row covering about $4 \%$ of the full range. 

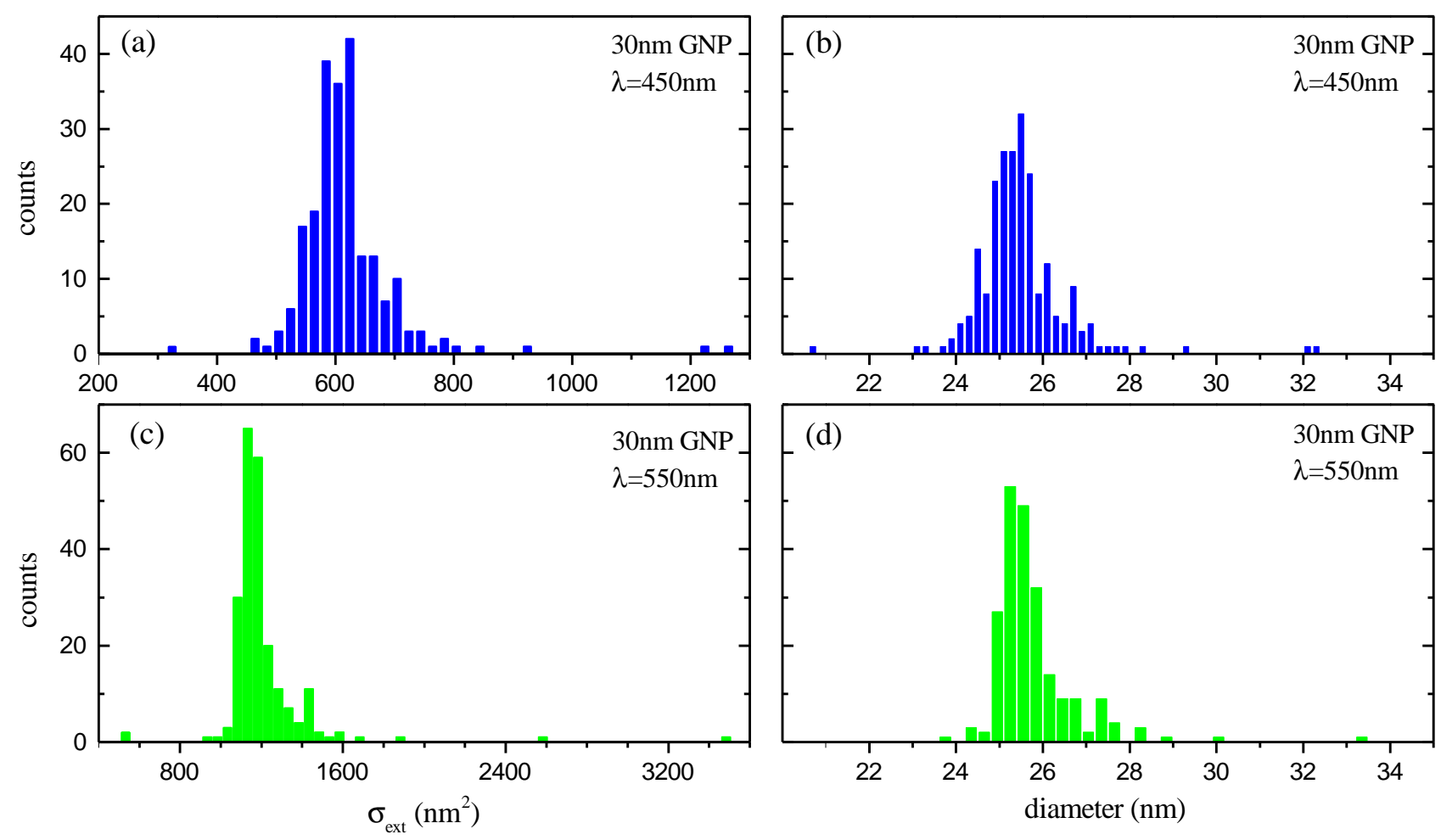

Figure 4. Measured cross-sections and deduced sizes of nominally $30 \mathrm{~nm}$ diameter GNPs. Histograms of measured $\sigma_{\text {ext }}$ for the filter range B (a) and diameter (b) deduced using Eq. 6. (c),(d) same as (a),(b) for filter range G.

seen is consistent with the calculated spatial coherence function. The extrapolated $\sigma_{\text {ext }}$ of this particle, which is given by the scattering cross-section only, is below $100 \mathrm{~nm}^{2}$, see for example the data using the $0-1.2 \mathrm{NA}$ range.

Let us now turn to the long shadow effect. As we have seen in Fig. 2, the measured $\sigma_{\text {ext }}$ (i.e. the limit value of $\sigma_{\text {ext }}$ for large $r_{\mathrm{i}}$, called in the following $\sigma^{\mathrm{m}}$ ) is increasing with increasing NA of illumination. This effect is due to the oblique illumination used in the measurements, resulting in a reference intensity measured in the sample plane which is smaller by a factor $\cos \left(\theta_{\mathrm{i}}\right)$, with the angle $\theta_{\mathrm{i}}$ of the illumination from the sample plane normal (see Fig. 5), than the plane-wave intensity of the illumination, for which the cross-sections $\sigma^{\mathrm{PW}}$ are defined. A derivation of the resulting long shadow factor $I_{\mathrm{LSF}}$ is given in Sec. A. For a homogeneous illumination intensity in the BFP of the condenser, and a particle cross-section independent of illumination direction, applicable to spherical particles in an isotropic environment, the factor is given by

$$
\sigma^{\mathrm{m}}=\frac{2}{\cos \underline{\theta}_{\mathrm{i}}+\cos \bar{\theta}_{\mathrm{i}}} \sigma^{\mathrm{PW}}
$$

with the minimum $\left(\underline{\theta}_{\mathrm{i}}\right)$ and maximum $\left(\bar{\theta}_{\mathrm{i}}\right)$ illumination angles. The factor between the measured cross-section $\sigma^{\mathrm{m}}$ and the standard plane-wave cross-section $\sigma^{\mathrm{PW}}$ is $I_{\mathrm{LSF}}$, which is used in Fig. $2 \mathrm{~b}$ and $\mathrm{d}$ to show the measured $\sigma_{\text {ext }}$ (blue circles) and the expected behaviour given as straight line. We find that the measurements deviate from the expected behaviour for large NA ranges (see for example the $0-1.34$ data indicated in Fig. 2b). This is due to the deviation of the real illumination intensity versus NA from the assumed constant intensity, showing a significant reduction towards higher NA, as discussed in the appendix Fig. 6. Using the recalculated long shadow factor for these real condenser properties, the measurements (red squares in Fig. 6b) are in good agreement with the expectation. We therefore conclude that the long shadow factor is experimentally observed, and needs to be considered for quantitative determination of cross-sections and their comparison with theory. 


\section{PARTICLE SIZING}

To determine the size of the particles, we employ the model of a spherical particle with diameter, $D \ll \lambda$, with $\lambda$ the vacuum wavelength of the incident light. In this case, the polarizability is expressed as,

$$
\alpha_{\mathrm{P}}=4 \pi \varepsilon_{0} R^{3} \frac{\varepsilon_{\mathrm{p}}-\varepsilon_{\mathrm{m}}}{\varepsilon_{\mathrm{p}}+2 \varepsilon_{\mathrm{m}}},
$$

with the particle radius, $R=D / 2$, the vacuum permittivity, $\varepsilon_{0}$, the complex permittivity inside the particle $\varepsilon_{\mathrm{p}}$, and the permittivity of the surrounding medium, $\varepsilon_{\mathrm{m}}$. Note that $\varepsilon_{\mathrm{p}}$ is a function of $\lambda$, and we use $\varepsilon_{\mathrm{m}}$ as constant over the visible range. The absorption and scattering cross-sections can then be found as ${ }^{8}$

$$
\sigma_{\mathrm{abs}}=\frac{k}{\varepsilon_{0}} \operatorname{Im} \alpha_{\mathrm{P}}, \quad \text { and } \quad \sigma_{\mathrm{sca}}=\frac{k^{4}}{\varepsilon_{0}}\left|\alpha_{\mathrm{P}}\right|,
$$

with the wavenumber, $k=2 \pi n / \lambda$, and the surrounding refractive index $n=\sqrt{\varepsilon_{\mathrm{m}}}$. For $D \approx 30 \mathrm{~nm}$ gold particles, $\sigma_{\text {sca }}<0.1 \sigma_{\text {ext }}$, so we can use the approximation $\sigma_{\text {ext }} \approx \sigma_{\text {abs }}$. To account for the wavelength range from $\lambda_{1}$ to $\lambda_{2}$ of the filter used, we calculate the average cross-section as

$$
\left\langle\sigma_{\mathrm{abs}}\right\rangle=D^{3} \frac{\pi^{2} n}{\left(\lambda_{2}-\lambda_{1}\right)} \int_{\lambda_{1}}^{\lambda_{2}} \operatorname{Im}\left(\frac{\varepsilon_{\mathrm{p}}-\varepsilon_{\mathrm{m}}}{\varepsilon_{\mathrm{p}}+2 \varepsilon_{\mathrm{m}}}\right) \frac{d \lambda}{\lambda} .
$$

and deduce the diameter $D$ by equating Eq. 6 to the measured cross-section corrected for the long shadow factor.

The measured cross-sections and the deduced diameter histograms for $I_{\mathrm{LSF}}=1.1$ are shown in Fig. 4 for 223 of nominally $30 \mathrm{~nm}$ GNPs analyzed with $r_{\mathrm{i}}=2.1 \mu \mathrm{m}$, both for the B and G filter range, and illumination range matched to 0.95 objective NA. We find that they provide consistent sizes of around $26 \mathrm{~nm}$, similar although slightly smaller than the diameter provided by the manufacturer from TEM analysis $(D=29.4 \pm 1.3 \mathrm{~nm})$. This could be due to the simplicity of the perfectly spherical particle model. Going beyond this model will be the subject of future work. To that end, in general, more complicated particle shapes can be calculated. For elliptical particles in the dipole limit, similar analytic expressions exist. ${ }^{6,8}$ For more complex shapes, and outside the dipole limit, numerical calculations can be used for optical sizing. ${ }^{9,10}$

\section{CONCLUSION}

Quantitative cross-section measurements at the single particle level, and comparison with simulations accurately modeling the measurement conditions, is still in its infancy. Indeed, previous reports of optical sizing based on the cross-section magnitude ${ }^{9,11}$ often highlight significant inconsistencies with data, or reach a good agreement by using model parameters which are not verified independently. Thanks to its operational simplicity and low cost, the method presented here has the potential to be widely adopted. In particular, high-throughput optical sizing via automated image analysis can provide an efficient and rapid all-optical structural characterization of a sample, reducing the need for more costly and time-consuming electron microscopy. In the present paper, we have demonstrated the long-shadow effect in cross-section measurements performed using transmission microscopy, which yields a systematic increase of the measured cross-section compared to its definition using plane waves. We believe that refining the quantitative method by identifying and eliminating systematic errors will offer an important characterization tool, and enable novel physical insights into the validity of theoretical models on the one hand, and the material properties on the other.

\section{APPENDIX A. LONG SHADOW EFFECT}

The sample is illuminated by a high NA condenser lens. Lens systems transform positions $\left(\rho_{\mathrm{i}}, \varphi_{\mathrm{i}}\right.$ in cylindrical coordinates) in the back focal plane (BFP) to directions $\left(\theta_{\mathrm{i}}, \varphi_{\mathrm{i}}\right.$ in spherical coordinates) impinging onto the front focal plane (FFP) and vice versa. The subscript i stands for "incidence" and denotes coordinates and quantities related to the illumination. We consider axially-symmetric optical elements only, so that $\varphi_{\mathrm{i}}$ is conserved in the 


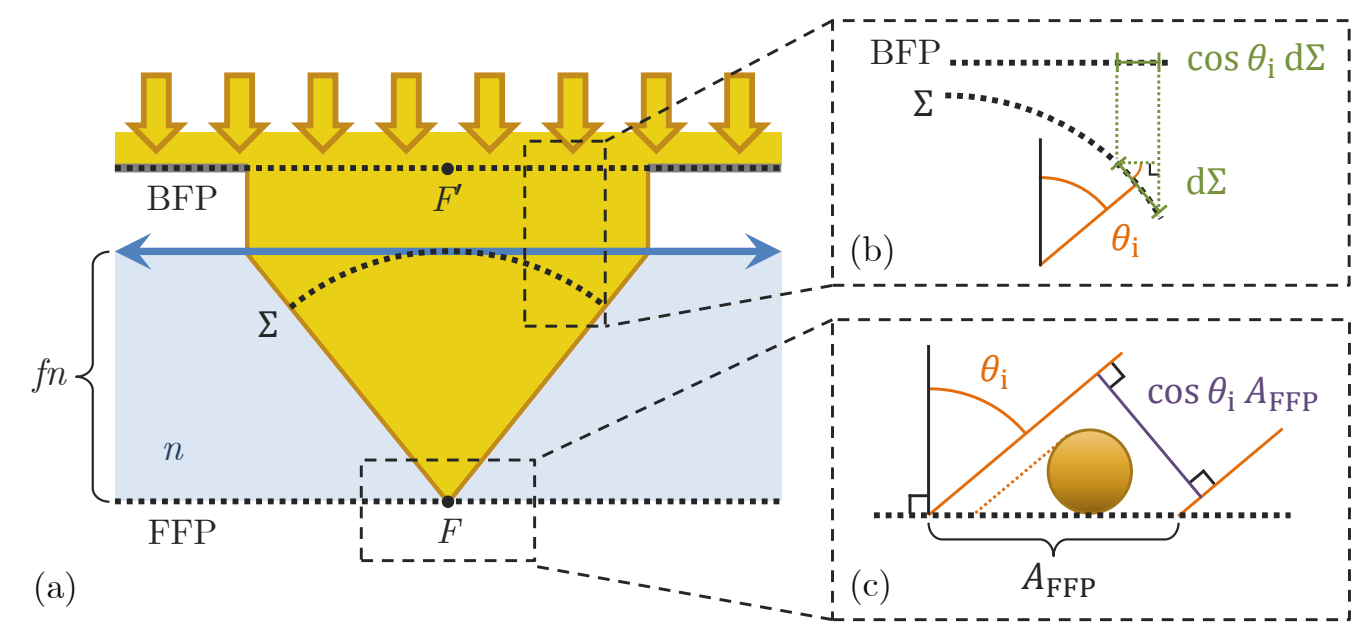

Figure 5. Sectional view of a typical Köhler illumination set-up for micro-spectroscopy experiments. The sketch depicts the main geometrical features associated with an aplanatic condenser lens (double arrow) discussed in the text. The angle of incidence $\theta_{\mathrm{i}}$ in the magnified insets (b) and (c) is chosen larger that the illumination range in (a) for clarity.

transformation. We assume that the condenser used is an aplanat, defined by a specific relation (Abbe's sine condition) between position $\rho_{\mathrm{i}}$ in the BFP and angle of incidence $\theta_{\mathrm{i}}$ in the FFP

$$
\rho_{\mathrm{i}}=f \mathrm{NA}_{\mathrm{i}}=f n \sin \theta_{\mathrm{i}}
$$

where $f$ is the effective focal length of the condenser, and $n$ is the refractive index of the medium filling the FFP.

From a geometric point of view, an aplanat maps the flat wavefront of a collimated beam in the BFP into a hemispherical surface $\Sigma$ (often called the front principal "plane") of radius $f n$ converging at the front focal point $F$ as depicted in Fig. 5a. In our case, the illumination originates from an incoherent source and therefore does not form a coherent wavefront in the BFP. Its coherence length in the BFP is given by the diffraction limit of the illuminated region in the FFP, which is an image of the field diaphragm in Köhler illumination. We use illuminated regions with sizes of about $300 \mu \mathrm{m}$, three orders of magnitude larger than the diffraction limited point spread function size for the maximum condenser NA. Accordingly, the coherence length in the BFP is about three orders of magnitude smaller than the size of the BFP. We can therefore treat the coherence areas in the BFP as small elements of area in the hemispherical surface $\mathrm{d} \Sigma=(f n)^{2} \mathrm{~d} \Omega_{\mathrm{i}}$ corresponding to a well-defined direction of incidence $\left(\theta_{\mathrm{i}}, \varphi_{\mathrm{i}}\right)$, of a plane wave propagating towards $F$. Note that the effective condenser focal length $f n$ is in our apparatus $10.5 \mathrm{~mm}$, much larger than the illuminated region, so that the illumination in the BFP of the condenser is still collimated to a small angular range, in our case about 0.03 radians. The illumination impinging on the sample is then the incoherent superposition of these plane waves (PWs). Now, the small element of area $\mathrm{d} A_{\mathrm{BFP}}$ corresponding to $\mathrm{d} \Sigma$ shrinks towards the edges of the BFP, as illustrated by the geometrical construction in Fig. 5b. Quantitatively, this result can be obtained as a consequence of the sine condition Eq. 7

$$
\mathrm{d} A_{\mathrm{BFP}}=\rho_{\mathrm{i}} \mathrm{d} \rho_{\mathrm{i}} \mathrm{d} \varphi_{\mathrm{i}}=(f n)^{2} \cos \theta_{\mathrm{i}} \sin \theta_{\mathrm{i}} \mathrm{d} \theta_{\mathrm{i}} \mathrm{d} \varphi_{\mathrm{i}}=(f n)^{2} \cos \theta_{\mathrm{i}} \mathrm{d} \Omega_{\mathrm{i}}=\cos \theta_{\mathrm{i}} \mathrm{d} \Sigma .
$$

The factor thereby introduced is sometimes referred to as the aplanatic apodization cosine. We want now to investigate the dependence on $\theta_{\mathrm{i}}$ of the power $\mathrm{d} P_{\mathrm{PW}}$ and intensity $\mathrm{d} I_{\mathrm{PW}}$ carried by the plane waves in our description. With $\mathrm{d} \Sigma$ and $\mathrm{d} A_{\mathrm{BFP}}$ related via Eq. 8, the same power crosses them by geometrical construction in a ray picture

$$
\mathrm{d} P_{\mathrm{PW}}=\mathrm{d} P_{\mathrm{BFP}}=I_{\mathrm{BFP}} \mathrm{d} A_{\mathrm{BFP}}=I_{\mathrm{BFP}} \cos \theta_{\mathrm{i}} \mathrm{d} \Sigma
$$

where $I_{\mathrm{BFP}}$ is the illumination intensity over the BFP, which is assumed to be constant. Eq. 9 shows that $\mathrm{d} P_{\mathrm{PW}}$ decreases as $\theta_{\mathrm{i}}$ increases, ruled by the aplanatic apodization cosine. 
Let us call $A_{\mathrm{FFP}}$ the illuminated region of the FFP. As discussed before, under the Köhler illumination scheme employed in our set-up, $A_{\mathrm{FFP}}$ is defined by the field aperture and is the same for all directions of incidence. This implies that the plane wave elements have a wavefront of size $A_{\mathrm{PW}}$, which according to the geometry given in Fig. $5 \mathrm{c}$ is given by $A_{\mathrm{PW}}=\cos \theta_{\mathrm{i}} A_{\mathrm{FFP}}$, reduced compared to $A_{\mathrm{FFP}}$ by the beam squeezing cosine. This expression of $A_{\mathrm{PW}}$ can be used to calculate the intensity of the plane wave

$$
\mathrm{d} I_{\mathrm{PW}} \equiv \frac{\mathrm{d} P_{\mathrm{PW}}}{A_{\mathrm{PW}}}=I_{\mathrm{BFP}} \frac{\mathrm{d} \Sigma}{A_{\mathrm{FFP}}}
$$

where the expression (9) of $\mathrm{d} P_{\mathrm{PW}}$ has been substituted in the last equality. Eq. 10 shows that $\mathrm{d} I_{\mathrm{PW}}$ is independent of the illumination direction: This is a non-trivial consequence of an exact compensation between the aplanatic apodization cosine and the beam squeezing cosine under the Köhler illumination scheme. Let us now recall the definition of the cross-section as the power removed from the exciting electromagnetic mode(s) by a given optical process divided by the incident intensity

$$
\sigma^{\mathrm{m}} \equiv \frac{P^{\mathrm{m}}}{I_{\mathrm{FFP}}}=\frac{\int_{\mathrm{ill}} \sigma^{\mathrm{PW}}\left(\theta_{\mathrm{i}}, \varphi_{\mathrm{i}}\right) \mathrm{d} I_{\mathrm{PW}}}{\int_{\mathrm{ill}} \cos \theta_{\mathrm{i}} \mathrm{d} I_{\mathrm{PW}}}
$$

Importantly, referencing occurs with respect to the intensity $I_{\mathrm{FFP}}$ traversing the FFP, to reproduce the way it is measured in experiments, where the microscope objective images the sample plane onto the sensor. In the second equality of Eq. 11, power and intensity are expressed as the sum over the contributions of the plane waves within the illumination cone. Note that while the removed power is proportional to the plane wave intensity $\mathrm{d} I_{\mathrm{PW}}$, the corresponding reference intensity is proportional to the power $\mathrm{d} P_{\mathrm{PW}}$ traversing $A_{\mathrm{FFP}}$, so that a $\cos \theta_{\mathrm{i}}$ factor appears at the denominator, see also Fig. 5c. Effectively, the way referencing is performed in the microscopy experiments results in $\sigma^{\mathrm{m}}>\sigma^{\mathrm{PW}}$, as if the particle shadow projected onto the FFP was measured (dashed orange line in Fig. 5c). By virtue of this analogy, we call the $\cos \theta_{\mathrm{i}}$ factor in Eq. 11 the long shadow cosine. $\mathrm{d} I_{\mathrm{PW}}$ given by Eq. 10 can be rewritten using Eq. 8 as

$$
\mathrm{d} I_{\mathrm{PW}}=\frac{I_{\mathrm{BFP}}}{A_{\mathrm{FFP}}} \frac{\mathrm{d} A_{\mathrm{BFP}}}{\cos \theta_{\mathrm{i}}}=\frac{I_{\mathrm{BFP}}}{A_{\mathrm{FFP}}}(f n)^{2} \mathrm{~d} \Omega_{\mathrm{i}}
$$

By substituting the first expression into Eq. 11

$$
\sigma^{\mathrm{m}}=\frac{\int_{A_{\mathrm{il}}}\left[\sigma^{\mathrm{PW}}\left(\theta_{\mathrm{i}}, \varphi_{\mathrm{i}}\right) / \cos \theta_{\mathrm{i}}\right] \mathrm{d} A_{\mathrm{BFP}}}{\int_{A_{\mathrm{il}}} \mathrm{d} A_{\mathrm{BFP}}}=\left\langle\frac{\sigma^{\mathrm{PW}}}{\cos \theta_{\mathrm{i}}}\right\rangle_{A_{\mathrm{i} 1}}
$$

we obtain a formula of simple interpretation: $\sigma^{\mathrm{m}}$ is the average of $\sigma^{\mathrm{Pw}}$ over the illuminated region $A_{\mathrm{il}}$ of the BFP, weighted by the inverse long shadow cosine to account for the experimental referencing to $I_{\mathrm{FFP}}$. Now, using $\sigma^{\mathrm{PW}}$ as a function of the direction of incidence, we recast Eq. 13 over the angular variables

$$
\sigma^{\mathrm{m}}=\frac{\int_{\Omega_{\mathrm{i}}} \sigma^{\mathrm{PW}}\left(\theta_{\mathrm{i}}, \varphi_{\mathrm{i}}\right) \mathrm{d} \Omega_{\mathrm{i}}}{\int_{\Omega_{\mathrm{i}}} \cos \theta_{\mathrm{i}} \mathrm{d} \Omega_{\mathrm{i}}}=\frac{\int_{\underline{\theta}_{\mathrm{i}}}^{\bar{\theta}_{\mathrm{i}}} \int_{0}^{2 \pi} \sigma^{\mathrm{PW}}\left(\theta_{\mathrm{i}}, \varphi_{\mathrm{i}}\right) \sin \theta_{\mathrm{i}} \mathrm{d} \theta_{\mathrm{i}} \mathrm{d} \varphi_{\mathrm{i}}}{\int_{\underline{\theta}_{\mathrm{i}}}^{\bar{\theta}_{\mathrm{i}}} \int_{0}^{2 \pi} \cos \theta_{\mathrm{i}} \sin \theta_{\mathrm{i}} \mathrm{d} \theta_{\mathrm{i}} \mathrm{d} \varphi_{\mathrm{i}}} .
$$

Somewhat surprisingly, due to the long shadow effect described above, $\sigma^{\mathrm{m}}$ is larger than $\sigma^{\mathrm{PW}}$ even in the most symmetric case of a sphere surrounded by a homogeneous medium. Indeed, this system is isotropic so that $\sigma^{\mathrm{PW}}$ does not depend on $\left(\theta_{\mathrm{i}}, \varphi_{\mathrm{i}}\right)$ and can be taken out of the integral (14), yielding

$$
\sigma^{\mathrm{m}}=\frac{2 \pi \sigma^{\mathrm{PW}} \int_{\underline{\theta}_{\mathrm{i}}}^{\bar{\theta}_{\mathrm{i}}} \sin \theta_{\mathrm{i}} \mathrm{d} \theta_{\mathrm{i}}}{2 \pi \int_{\underline{\theta}_{\mathrm{i}}}^{\bar{\theta}_{\mathrm{i}}} \cos \theta_{\mathrm{i}} \sin \theta_{\mathrm{i}} \mathrm{d} \theta_{\mathrm{i}}}=\frac{2 \sigma^{\mathrm{PW}}}{\cos \underline{\theta}_{\mathrm{i}}+\cos \bar{\theta}_{\mathrm{i}}} .
$$

Note that we had assumed that in the BFP of the condenser the intensity is constant, and that the condenser is ideal, i.e. its transmission does not depend on the BFP position. For real systems, both conditions are not 

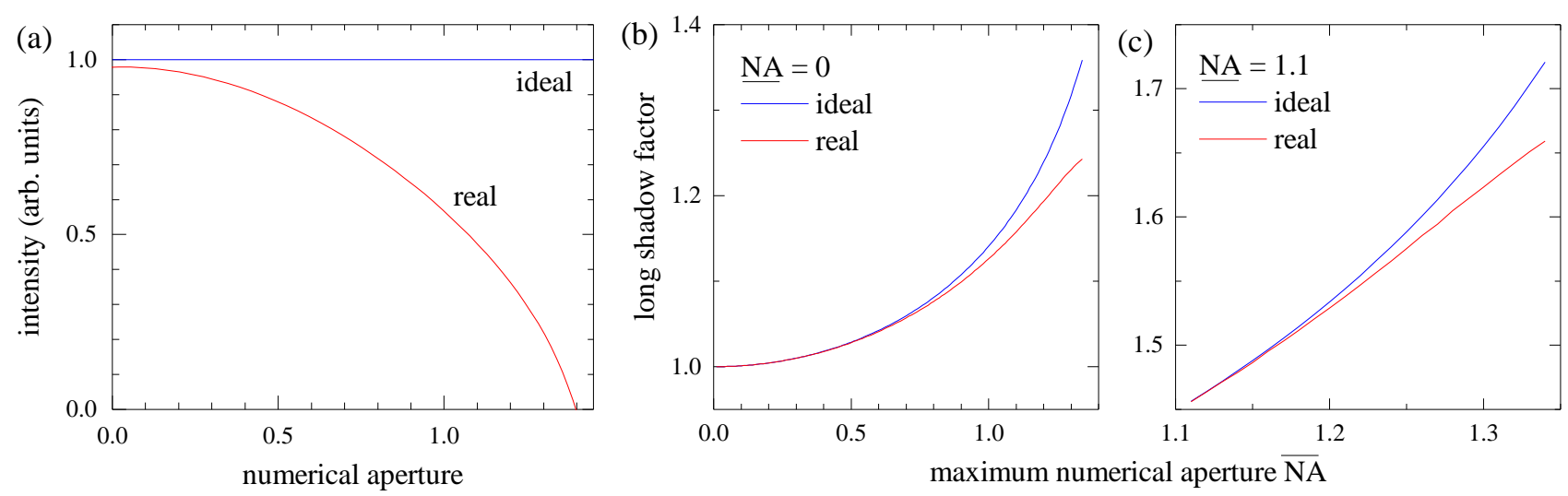

Figure 6. (a) Intensity transmitted by the condenser as function of the input $\mathrm{NA}_{\mathrm{i}}$, comparing measured (red) versus ideal (blue) case (see text). The corresponding long shadow factors are shown in (b) and (c) for the NA used in the experiments.

exactly fulfilled. We have therefore measured the transmitted intensity as function of the input NA, and fitted it with a suited function, as given in Fig. 6a, see details in the supplemental material of Ref. 10. We find that the intensity significantly decreases with increasing NA, mostly due to larger reflection losses at the edges of the optical elements constituting the condenser. Taking this into account in the integration of Eq. 13 results in a real long shadow factor, which is shown in Fig. $6 \mathrm{~b}$ together with the ideal long shadow factor for $\mathrm{NA}=0$, and in Fig. 6c for $\mathrm{NA}=1.1$, covering the two cases (BF and DF configurations) used in our experiments. We find the long shadow factor to be reduced taking into account the real condenser characteristics which are tantamount to a reduced contribution of high NAs.

\section{ACKNOWLEDGEMENTS}

We acknowledge funding from the Welsh Government Life Sciences Bridging Fund (grant LSBF/R6-005) and the European Commission, Research Executive Agency (Marie Curie Action 607842 FINON ITN-2013). YW acknowledges the BBSRC for his industrial CASE PhD studentship award (grant n. BB/L015889/1). PB acknowledges the Royal Society for her Wolfson research merit award (grant WM140077).

\section{REFERENCES}

[1] Talapin, D. V., Lee, J.-S., Kovalenko, M. V., and Shevchenko, E. V., "Prospects of colloidal nanocrystals for electronic and optoelectronic applications," Chem. Rev. 110, 389-458 (2010).

[2] Sun, T., Zhang, Y. S., Pang, B., Hyun, D. C., Yang, M., and Xia, Y., "Engineered nanoparticles for drug delivery in cancer therapy," Angew. Chem. Int. Ed. 53, 12320-12364 (2014).

[3] Sönnichsen, C., Reinhard, B. M., Liphardt, J., and Alivisatos, A. P., "A molecular ruler based on plasmon coupling of single gold and silver nanoparticles," Nat. Biotech. 23, 741-745 (2005).

[4] Mie, G., "Beiträge zur Optik trüber Medien, speziell kolloidaler Metallösungen," Ann. Phys. 330, 377-445 (1908).

[5] Payne, L. M., Langbein, W., and Borri, P., "Polarization-resolved extinction and scattering cross-section of individual gold nanoparticles measured by wide-field microscopy on a large ensemble," Appl. Phys. Lett. 102, 131107-1 (2013).

[6] Payne, L., Zoriniants, G., Masia, F., Arkill, K. P., Verkade, P., Rowles, D., Langbein, W., and Borri, P., "Optical micro-spectroscopy of single metallic nanoparticles: quantitative extinction and transient resonant four-wave mixing," Faraday Discuss. 184, 305-320 (2015).

[7] Payne, L. M., Langbein, W., and Borri, P., "Wide-field imaging of single-nanoparticle extinction with sub-nm² sensitivity," Phys. Rev. Applied 9, 034006 (2018).

[8] Bohren, C. F. and Huffman, D. R., Absorption and scattering of light by small particles, John Wiley \& Sons, New York (1983). 
[9] Davletshin, Y. R., Lombardi, A., Cardinal, M. F., Juvé, V., Crut, A., Maioli, P., Liz-Marzán, L. M., Vallée, F., Del Fatti, N., and Kumaradas, J. C., "A quantitative study of the environmental effects on the optical response of gold nanorods," ACS Nano 6(9), 8183-8193 (2012).

[10] Zilli, A., Langbein, W., and Borri, P., "Lights and shadows at the nanoscale: Measuring the optical crosssections of single nano-objects," Submitted (2019).

[11] Muskens, O. L., Billaud, P., Broyer, M., Fatti, N. D., and Vallée, F., "Optical extinction spectrum of a single metal nanoparticle: Quantitative characterization of a particle and of its local environment," Phys. Rev. B 78, 205410 (2008). 